University of Nebraska - Lincoln

DigitalCommons@University of Nebraska - Lincoln

2-15-1999

\title{
Thermodynamic expansion of nucleation free-energy barrier and size of critical nucleus near the vapor-liquid coexistence
}

\author{
Kenichiro Koga \\ University of Nebraska-Lincoln, koga@cc.okayama-u.ac.jp \\ Xiao Cheng Zeng \\ University of Nebraska-Lincoln, xzeng1@unl.edu
}

Follow this and additional works at: https://digitalcommons.unl.edu/chemzeng

Part of the Chemistry Commons

Koga, Kenichiro and Zeng, Xiao Cheng, "Thermodynamic expansion of nucleation free-energy barrier and size of critical nucleus near the vapor-liquid coexistence" (1999). Xiao Cheng Zeng Publications. 62. https://digitalcommons.unl.edu/chemzeng/62

This Article is brought to you for free and open access by the Published Research - Department of Chemistry at DigitalCommons@University of Nebraska - Lincoln. It has been accepted for inclusion in Xiao Cheng Zeng Publications by an authorized administrator of DigitalCommons@University of Nebraska - Lincoln. 


\title{
Thermodynamic expansion of nucleation free-energy barrier and size of critical nucleus near the vapor-liquid coexistence
}

\author{
Kenichiro Koga ${ }^{\mathrm{a})}$ and X. C. Zeng \\ Department of Chemistry and Center for Materials Research and Analysis, University of Nebraska-Lincoln, \\ Lincoln, Nebraska 68588
}

(Received 28 September 1998; accepted 10 November 1998)

\begin{abstract}
Nucleation free-energy barrier height and size of the critical nucleus are expanded in powers of the chemical potential difference between the supersaturated vapor (or expanded liquid) in the metastable state and the saturated vapor-liquid system in the stable equilibrium state at the same temperature. The coefficients in the expansions are expressed in terms of the thermodynamic properties at the stable equilibrium state. Comparisons with the results obtained from the density-functional calculation for nucleation of the Lennard-Jones fluid show that systematic improvement in predicting properties of the critical nucleus, either liquid droplet or vapor cavity, is achieved by adding the higher order terms in the expansions. The scaling relations proposed by McGraw and Laaksonen are found to be good approximations to the general expansion; in particular, the barrier height displacement appearing in these scaling relations is naturally given as the second order coefficient in the expansion of the barrier height. (C) 1999 American Institute of Physics. [S0021-9606(99)50707-1]
\end{abstract}

\section{INTRODUCTION}

A central concern in the development of theory of nucleation, either a phenomenological one or a molecular-based one, is the nucleation free-energy barrier height $W^{*}$, for this quantity primarily controls the rate of nucleation. ${ }^{1}$ The capillary drop model in the classical nucleation theory ${ }^{2}$ (CNT), for example, expresses the barrier height in terms of several measurable macroscopic properties including the tension of planner vapor-liquid interface. The latter quantity is assumed to be the same as the surface tension of the critical nucleus; in other word, the Tolman length is assumed to be zero in the classical nucleation theory. Modern theory of nucleation, such as the $i / v$ cluster theory and its derivative by Reiss and co-workers ${ }^{3}$ or the density-functional (DF) theory of nucleation by Oxtoby and co-workers, ${ }^{4-6}$ offers a molecular-based approach to obtain $W^{*}$ (the Tolman length can be given from DF approach as well). These theories reveal that the assumptions underlying the CNT are, in certain circumstances, invalid. In turn, these molecular-based theories stimulated new efforts in searching for better phenomenological theories of nucleation. ${ }^{7-9}$ Recently, McGraw and Laaksonen ${ }^{7}$ proposed a set of scaling relations for the size of critical nucleus $\Delta n^{*}$, the nucleation barrier height $W^{*}$ and their combination. According to their scaling relation the true barrier height $W^{*}$ differs from the CNT prediction $W_{\mathrm{CNT}}^{*}$ by a function only of temperature $D(T)$ (also called the barrier height displacement), that is, the difference $W^{*}-W_{\mathrm{CNT}}^{*}$ depends neither on the nucleus size nor the supersaturation ratio $S$ of vapor. Within the framework of DF approach, they confirmed that $W^{*}-W_{\mathrm{CNT}}^{*}$ only changes a few percent at various supersaturations while the barrier height itself varies by an order of magnitude. In a later ar-

a)Electronic mail: koga@super2.unl.edu ticle, McGraw and Laaksonen ${ }^{8}$ discussed the physical basis for the barrier height displacement $D(T)$ using a nonuniform spherical droplet model of the nucleus.

The advanced scaling relations by McGraw and Laaksonen have made an important step towards developing better phenomenological theory of nucleation for describing experiments. Talanquer proposed a simple method to determine $D(T)$, that is, $D(T)$ can be obtained by evaluating $W_{\mathrm{CNT}}^{*}$ for the critical nucleus at the spinodal. The spinodal is calculated from the equation of state, obtained by fitting the experimental values of vapor pressure and binodal curve. Talanquer found that the McGraw-Laaksonen scaling relations improve the description of homogeneous nucleation for both nonpolar and weakly polar systems. It seems that McGrawLaaksonen's scaling relations indeed provide a simple explanation for reported systematic discrepancies between CNT and measurements. ${ }^{10}$

However, it is fair to mention that the McGrawLaaksonen scaling relations have been obtained as homogeneous solutions to a differential equation derived from the nucleation theorem (21) and a general form for $W^{*} .{ }^{7,11}$ Since the homogeneity ansatz has not been proved in general, it remains unanswered why the scaling relations of McGraw and Laaksonen are overall successful. Furthermore, the following two questions also need to be addressed: Can the scaling relations be derived and justified based on a more rigorous theoretical foundation? Can the barrier height displacement $D(T)$ be given in terms of thermodynamic properties at stable equilibrium state, rather than determined based on the spinodal condition? In this regard, it is important to pursue the derivation of $W^{*}$ and $\Delta n^{*}$ rigorously based on the Gibbs theory of capillarity. In this article we present thermodynamic expansions of $W^{*}$ and $\Delta n^{*}$ in powers of the chemical potential difference $\Delta \mu$ between the su- 
persaturated and saturated vapor at a given temperature. We show that the McGraw-Laaksonen scaling relations are recovered by considering only the second-order term in the expansion of $W^{*}$ as a correction to $W_{\mathrm{CNT}}^{*}$. The supersaturation-independent displacement $D(T)$ is in fact just the coefficient of the second-order term. The coefficients in the expansion including $D(T)$ are expressed in terms of the bulk and surface properties at the stable equilibrium state of vapor-liquid coexistence. Among others, these properties include the Tolman length ${ }^{12}$ and the fundamental length characteristic of liquid proposed by Egelstaff and Widom. ${ }^{13}$

In the next section, we derive the expansions of thermodynamic formulas for the nucleation barrier height $W^{*}$ and the size of critical nucleus $\Delta n^{*}$. In Sec. III, we examine the effects of truncation of the higher order terms in the expansions within the framework of the density functional theory of vapor-liquid nucleation. Our concluding remarks are given in Sec. IV.

\section{EXPANSIONS OF NUCLEATION FREE-ENERGY BARRIER HEIGHT AND THE SIZE OF CRITICAL NUCLEUS IN POWERS OF THE CHEMICAL POTENTIAL}

We consider vapor-to-liquid and liquid-to-vapor nucleation of a single component fluid under a condition of fixed temperature $T$. Here the nucleation barrier height $W^{*}$, which is the work of formation of the critical nucleus, and the size of the nucleus $\Delta n^{*}$, which is the excess number of molecules in the critical nucleus over that present in the same volume in the surrounding bulk phase, are considered as functions only of the chemical potential $\mu$ of the system or the difference $\Delta \mu$ from that of the saturated vapor. In this section we show that $W^{*}$ and $\Delta n^{*}$ can be expanded around $\Delta \mu=0$ and that the coefficients in the expansion are expressed in terms of the bulk and surface properties at the stable equilibrium state.

\section{A. Nucleation free-energy barrier height}

We start from the formally exact expression for the nucleation free-energy barrier height (or the work of formation) of the spherical critical nucleus ${ }^{14}$

$$
W^{*}=\frac{16 \pi \sigma_{\mathrm{s}}^{3}}{3(\Delta p)^{2}}
$$

where $\sigma_{\mathrm{s}}$ is the surface tension with respect to the surface of tension and $\Delta p \equiv p^{\alpha}-p^{\beta}$ is the pressure difference between the homogeneous liquid $(\alpha)$ and vapor phase $(\beta)$ having the same chemical potential; in the case of vapor-to-liquid nucleation $(\Delta \mu>0)$, phase $\alpha$ is thermodynamically stable whereas phase $\beta$ is metastable. Note that Eq. (1) is valid up to the smallest critical nucleus. It is clear from Eq. (1) that $W^{*}$, a function of $\Delta \mu$, has the pole of second order at $\Delta \mu$ $=0$. Therefore a function of $\Delta \mu$ defined by

$$
g=W^{*}(\Delta \mu)^{2}
$$

does not diverge at $\Delta \mu=0$. In general this function can be expanded as

$$
g(x)=\frac{1}{2} C+B x-D x^{2}-\sum_{i=1}^{\infty} \frac{A_{i}}{i} x^{i+2},
$$

where $x=\Delta \mu$ and

$$
\begin{aligned}
& C=2 g(0), \\
& B=g^{\prime}(0), \\
& D=-\frac{1}{2} g^{\prime \prime}(0), \\
& A_{i}=-\frac{i}{(i+2) !} g^{(i+2)}(0),
\end{aligned}
$$

where ', ", and ${ }^{(n)}$ stand for, respectively, the first, second, and $n$th derivatives with respect to $x(=\Delta \mu)$. Once these coefficients are obtained in terms of thermodynamic properties at the vapor-liquid coexistence, the nucleation freeenergy barrier height is given as an explicit function of $\Delta \mu$ :

$$
W^{*}=\frac{1}{2} C(\Delta \mu)^{-2}+B(\Delta \mu)^{-1}-D-\sum_{i=1}^{\infty} \frac{A_{i}}{i}(\Delta \mu)^{i} \text {. }
$$

Systematic calculation of the coefficients can be carried out in the following manner. Let $y(x)=\Delta p$ and $z(x)$ $=y(x) / x$ for convenience. Then $g(x)$ is rewritten as

$$
g(x)=\frac{16 \pi \sigma_{\mathrm{s}}^{3}}{3 z^{2}}
$$

and in general the $n$th derivative $g^{(n)}(x)$ is expressed in terms of $\sigma_{\mathrm{s}}, \sigma_{\mathrm{s}}^{\prime}, \ldots, \sigma_{\mathrm{s}}^{(n)}$ and $z, z^{\prime}, \ldots, z^{(n)}$. When we calculate the limits of $g(x), g^{\prime}(x), \ldots, g^{(n)}(x)$ at $x=0$, we use the following relations (10)-(17): First, since $z=y(x) / x$ and $y(0)=0$ the limits of the derivatives of $z$ and of $y$ at $x=0$ are related by the mathematical relation:

$$
\lim _{x \rightarrow 0} z^{(n)}=y^{(n+1)}(0) /(n+1),
$$

including the case $n=0$. Second, we have the following thermodynamic relations for the bulk properties:

$$
\begin{aligned}
& y^{\prime}=\left(\frac{\partial \Delta p}{\partial \mu}\right)_{T}=\Delta \rho, \\
& y^{\prime \prime}=\left(\frac{\partial^{2} \Delta p}{\partial \mu^{2}}\right)_{T}=\Delta \rho^{2} \chi, \\
& y^{(n+2)}=\left(\frac{\partial^{n+2} \Delta p}{\partial \mu^{n+2}}\right)_{T}=\left(\Delta \rho^{2} \chi\right)^{(n)},
\end{aligned}
$$

where $\Delta \rho=\rho^{\alpha}-\rho^{\beta}$ is the density difference between the two homogeneous phases and $\Delta \rho^{2} \chi=\left(\rho^{\alpha}\right)^{2} \chi^{\alpha}-\left(\rho^{\beta}\right)^{2} \chi^{\beta}$ is the difference in the product of square of density $\left(\rho^{2}\right)$ and isothermal compressibility $(\chi)$ between the two phases. Third, we have a thermodynamic relation for the surface properties,

$$
\lim _{x \rightarrow 0} \sigma_{\mathrm{s}}^{\prime} / \Delta \rho=-\delta_{\infty},
$$

where $\delta_{\infty}$ is the Tolman length originally defined as the limit of distance from the surface of tension to the equimolar di- 
viding surface as $x \rightarrow 0 .{ }^{12}$ Equation (14) stems from two relations about the adsorption at the surface of tension: ${ }^{12,14,15}$

(i) $\Gamma_{s}=-\left(\partial \sigma_{\mathrm{s}} / \partial \mu\right)_{T}$ and (ii) $\delta_{\infty}=\lim _{\Delta \mu \rightarrow 0} \Gamma_{s} / \Delta \rho$. Hereinafter, we denote the surface tension and its derivatives at $x$ $=0$ [except the first derivative replaced by Eq. (14)] as $\sigma$, $\sigma^{\prime \prime}$, etc. without the subscript s.

If we calculate the limits of $g(x), g^{\prime}(x), \ldots, g^{(n)}(x)$ as $x \rightarrow 0$ using the relations (10)-(14) and then substitute the limits into Eqs. (4)-(7), we obtain the desired thermodynamic expressions for the coefficients of the expansion of the nucleation barrier height. The first five coefficients are given as

$$
C=\frac{32 \pi \sigma^{3}}{3(\Delta \rho)^{2}}
$$

$$
\begin{aligned}
B= & -\frac{16 \pi \sigma^{2}}{\Delta \rho}\left[\delta_{\infty}+\frac{1}{3} \Delta_{\infty}\right], \\
D= & -16 \pi \sigma\left\{\left[\delta_{\infty}+\frac{1}{2} \Delta_{\infty}\right]^{2}\right. \\
& \left.+\frac{\sigma^{2}}{2(\Delta \rho)^{2}}\left[\frac{\sigma^{\prime \prime}}{\sigma}-\frac{2\left(\Delta \rho^{2} \chi\right)^{\prime}}{9 \Delta \rho}\right]\right\}, \\
A_{1}= & \frac{16 \pi}{3}\left\{\Delta \rho\left[\delta_{\infty}+2 \Delta_{\infty}\right]\left[\delta_{\infty}+\frac{1}{2} \Delta_{\infty}\right]^{2}\right. \\
& \left.+\frac{3 \sigma^{2}\left[\frac{\sigma^{\prime \prime}}{\Delta \rho}-\frac{\left(\Delta \rho^{2} \chi\right)^{\prime}}{3 \Delta \rho}\right]\left[\delta_{\infty}+\frac{1}{2} \Delta_{\infty}\right]}{}+\frac{\sigma^{3}}{2(\Delta \rho)^{2}}\left[\frac{\sigma^{\prime \prime \prime}}{\sigma}-\frac{\left(\Delta \rho^{2} \chi\right)^{\prime \prime}}{6 \Delta \rho}\right]\right\},
\end{aligned}
$$

where the quantity $\Delta_{\infty}$ is defined as

$$
\Delta_{\infty}=\frac{\sigma \Delta \rho^{2} \chi}{(\Delta \rho)^{2}}
$$

which has a dimension of length. Note that near the triple point, $\rho^{\alpha} \gg \rho^{\beta}$ and $\left(\rho^{\alpha}\right)^{2} \chi^{\alpha} \gg\left(\rho^{\beta}\right)^{2} \chi^{\beta}$; therefore $\Delta_{\infty} \approx \sigma \chi^{\alpha}$, which is the fundamental length characteristic of the liquid proposed by Egelstaff and Widom. ${ }^{13}$ The quantities in Eqs. (15)-(20) are all defined at the stable equilibrium state of vapor-liquid coexistence $(\Delta \mu=0)$; the coefficients are therefore functions only of $T$.

Since $W^{*}$ is expressed in terms of the bulk property $(\Delta p)$ and the surface property $(\sigma)$ of the system, the coefficients of the expansion about $\Delta \mu=0$ consist of the derivatives of the bulk and surface properties with respect to chemical potential at $\Delta \mu=0$. For example, expression of the coefficient $C$ includes the first derivative of the bulk pressure as $\Delta \rho$ whereas the coefficient $B$ contains the derivative of the surface tension as $-\delta_{\infty} \Delta \rho$ and the second derivative of pressure as $\Delta \rho^{2} \chi$. In general a higher-order term's coefficient has higher-order derivatives of the bulk pressure and surface tension with respect to the chemical potential.

\section{B. Expansion of the size of critical nucleus}

The size of critical nucleus $\Delta n^{*}$ is defined as the excess number of molecules in the critical nucleus over that present in the same volume in the homogeneous phase surrounding the nucleus. Unlike the number of molecules in the critical nucleus defined by introducing a dividing surface, the excess $\Delta n^{*}$ is independent of the choice of the dividing surface. The nucleation theorem ${ }^{16-18}$ gives a relationship between $\Delta n^{*}$ and $W^{*}$,

$$
\left(\frac{\partial W^{*}}{\partial \mu}\right)_{T}=-\Delta n^{*} \text {. }
$$

Note that Eq. (21) is a general thermodynamic relation as proved by Oxtoby and Kashchiev. ${ }^{17}$ From the nucleation theorem (21), the expansion of $\Delta n^{*}$ is immediately obtained from differentiating Eq. (8) with respect to $\Delta \mu$ as:

$$
\Delta n^{*}=C(\Delta \mu)^{-3}+B(\Delta \mu)^{-2}+\sum_{i=1}^{\infty} A_{i}(\Delta \mu)^{i-1} .
$$

Note also that the term $(\Delta \mu)^{-1}$ does not exist in the expansion.

Expanding $f(x) \equiv \Delta n^{*} x^{3}=-W^{* \prime} x^{3}$ as done for $g(x)$, the same coefficients for $\Delta n^{*}$ are obtained. In particular, we find $f^{\prime \prime}(0)=0$, which is consistent with that the term $(\Delta \mu)^{-1}$ does not exist in Eq. (22). It should be noted, however, that the thermodynamic expression (17) for the coefficient $D$ cannot be obtained from $\Delta n^{*}$ [Eq. (22)] to $W^{*}[$ Eq. (8)] by integration.

\section{Relation with other phenomenological approaches}

The expansions in Eqs. (8) and (22) for $W^{*}$ and $\Delta n^{*}$ are general thermodynamic results, for they are derived based solely on the Gibbs theory of capillarity and the nucleation 
theorem. The expansions are analogous to the virial expansion of the equation of state. One can expect better results as more higher order terms are used. In fact, we found that the classical expressions for the barrier height and the size of critical nucleus and the scaling relations by McGraw and Laaksonen are special forms of the general results.

First, the classical expression for the nucleation barrier height is recovered if $B=0, D=0, A_{i}=0$ for all $i$ :

$$
W_{\mathrm{CNT}}^{*}=\frac{1}{2} C(\Delta \mu)^{-2},
$$

where $C$ is given by Eq. (15). ( $\Delta \rho$ in $C$ is usually replaced by $\rho^{\alpha}$, which is reasonable near the triple point.) Under the same condition, the classical expression for $\Delta n^{*}$ (the Kelvin relation) is also recovered:

$$
\Delta n_{\mathrm{CNT}}^{*}=C(\Delta \mu)^{-3} \text {. }
$$

Therefore, the capillary drop model in CNT is the zeroth order approximation to the general expansions, which is only exact in the limit as $\Delta \mu \rightarrow 0$. This is analogous to the ideal gas approximation to the virial expansion of the equation of state.

Second, the McGraw and Laaksonen scaling relations ${ }^{7}$ are recovered if $B=0$ and $A_{i}=0$ for all $i$. The scaling relation for $\Delta n^{*}$ is the same as the classical expression (24). From the nucleation theorem (21), integrating Eq. (24) with respect to $\Delta \mu$ gives rise to the scaling relation for $W^{*}$. Here, $-D$ appears as an integration constant although $D$ does not exist in the CNT expression (23). Furthermore, combining the two scaling relations for $\Delta n^{*}$ and $W^{*}$ results in the scaling relation for $W^{*} / \Delta n^{*} \Delta \mu$. It has been shown ${ }^{7}$ that results of $W^{*}$ and $\Delta n^{*}$ obtained from DF calculation follow the scaling relations over a wide range of $\Delta \mu$ if $D$ is determined by the average displacement $W_{\mathrm{CNT}}^{*}-W^{*}$. Recently, Talanquer ${ }^{9}$ proposed that the constant $D$ is determined from the boundary condition that $W^{*}=0$ at spinodal. However, since $B \neq 0$ and $A_{i} \neq 0$ in general, the McGraw-Laaksonen scaling relations are basically particular approximations to the general expansions. In principle, $D$ should be determined by Eq. (17). The reason why the McGraw-Laaksonen scaling relations are successful for a wide range of $\Delta \mu$ will be discussed in the next section.

\section{NUMERICAL RESULTS AND DISCUSSION}

In this section, the analytical results in Sec. II are examined by numerical calculation of the Lennard-Jones model fluid based on the density-functional theory of nucleation., The details of the DF approach to the LJ fluid nucleation have been given elsewhere. ${ }^{5}$ The properties of the critical nucleus are computed over a wide range of $\Delta \mu$ (from -1.2 to 1.8 in reduced units) at a fixed temperature $T$ near the triple point $\left(T^{*}=0.7\right.$ in reduced units, corresponding in the case of argon to $T=84 \mathrm{~K}$ ). Note that the negative $\Delta \mu$ corresponds to the liquid-to-vapor nucleation or bubble formation.

Determination of the coefficients in the expansion requires the bulk and interfacial properties at the stable equilibrium state $(\Delta \mu=0)$. The bulk properties such as $\Delta \rho^{2} \chi$ and higher derivatives of $\Delta \rho^{2} \chi$ with respect to $\Delta \mu$ are
TABLE I. Thermodynamic properties at stable equilibrium of vapor-liquid coexistence determining the coefficients in the thermodynamic expansions of the nucleation free-energy barrier height and the size of critical nucleus. The primes denotes derivatives with respect to chemical potential at fixed temperature. The values are obtained from the density functional approach to nucleation of the Lennard-Jones fluid. See the text for details. Temperature is 0.7 in reduced units. All the values are given in reduced units.

\begin{tabular}{crcrlr}
\hline \hline Coefficient & Value & Property & Value & Property & Value \\
\hline$C$ & 100.15 & $\sigma$ & 1.287 & $\Delta \rho$ & 0.844 \\
$B$ & 11.35 & $\sigma^{\prime}$ & 0.131 & $\Delta \rho^{2} \chi$ & 0.066 \\
$D$ & 29.00 & $\sigma^{\prime \prime}$ & -0.520 & $\left(\Delta \rho^{2} \chi\right)^{\prime}$ & -0.038 \\
$A_{1}$ & $-1.5^{\mathrm{a}}$ & $\sigma^{\prime \prime \prime}$ & 0.272 & $\left(\Delta \rho^{2} \chi\right)^{\prime \prime}$ & 0.038 \\
$A_{2}$ & $-69.2^{\mathrm{a}}$ & $\sigma^{\prime \prime \prime \prime}$ & 5.784 & $\left(\Delta \rho^{2} \chi\right)^{\prime \prime \prime}$ & -0.091 \\
& & $\delta_{\infty}$ & -0.155 & $\Delta_{\infty}$ & 0.120 \\
\hline \hline
\end{tabular}

axpected numerical errors are about \pm 1 for $A_{1}$ and \pm 40 for $A_{2}$ due to the uncertainty in determining $\sigma^{\prime \prime \prime}$ and $\sigma^{\prime \prime \prime \prime}$.

obtained from the equation of state for $\mu(\rho, T)$ of the model fluid $^{5}$ through the thermodynamic relations: $\rho^{2} \chi$ $=(\partial \mu / \partial \rho)^{-1},\left(\rho^{2} \chi\right)^{\prime}=-\left(\partial^{2} \mu / \partial \rho^{2}\right)(\partial \mu / \partial \rho)^{-3}$, etc. Calculation of the surface properties requires the density profile of the inhomogeneous system. The surface tension $\sigma$ is obtained by DF calculation of the planner interface. The derivatives of surface tension $\sigma^{\prime}, \sigma^{\prime \prime}, \sigma^{\prime \prime \prime}$, and $\sigma^{\prime \prime \prime \prime}$ are evaluated by fitting a polynomial to a set of data $\left(\Delta \mu, \sigma_{s}\right) .{ }^{19}$ The Tolman length $\delta_{\infty}$ is determined from $\sigma^{\prime}$ (the adsorption route $^{20}$ to $\delta_{\infty}$ ). Substituting these results into Eqs. (15)-(19) gives the coefficients. Table I summarizes the coefficients together with the properties determining the coefficients. The Tolman length $\delta_{\infty}$ and the fundamental length $\Delta_{\infty}$ characteristic of the liquid are comparable in magnitude and opposite in sign. ${ }^{21}$ Therefore the sum $\left[\delta_{\infty}+k \Delta_{\infty}\right]$ with the factor $k$ of order of 1 is very small. Thus, the coefficient $B$ containing the sum is much smaller than $C$. For the same reason, the terms having $\left[\delta_{\infty}+k \Delta_{\infty}\right]$ in Eqs. (17)-(19) are negligible for determining the coefficients $D, A_{1}$, and $A_{2}$. The coefficient $D$ is primarily determined by the term having $\sigma^{\prime \prime}$ in Eq. (17), coefficient $A_{1}$ by $\sigma^{\prime \prime \prime}$ in Eq. (18), and coefficient $A_{2}$ by $\sigma^{\prime \prime \prime \prime}$ in Eq. (19).

The expansion formula in Eq. (8) for $W^{*}$ is examined through comparisons with results obtained from DF calculation for the LJ fluid model. The quantity $2 W^{*}(\Delta \mu)^{2} / C$ is plotted in Fig. 1 against $\Delta \mu$ in reduced units. The horizontal lines in Fig. 1 correspond to prediction of the classical nucleation theory. The zeroth order approximation works better in the case of vapor-to-liquid nucleation [Fig. 1(a)] compared to liquid-to-vapor nucleation [Fig. 1(b)] due to the asymmetry in $2 W^{*}(\Delta \mu)^{2} / C$. The first-order correction to CNT by introducing the linear term with coefficient $B$ gives the correct slope at $\Delta \mu=0$; however the range of its validity is as limited as that of CNT. Inclusion of the second-order term with coefficient $D$ makes significant improvement to the firstorder approximation. Because of the parabola-like nature of $2 W^{*}(\Delta \mu)^{2} / C$, the prediction of the second-order approximation agrees qualitatively well with the result obtained from DF calculation over a whole range of $\Delta \mu$. The thirdorder approximation by including the cubic term with coefficient $A_{1}$ improves the second-order prediction little, because $A_{1}$ is much smaller than $D$ when compared in reduced 

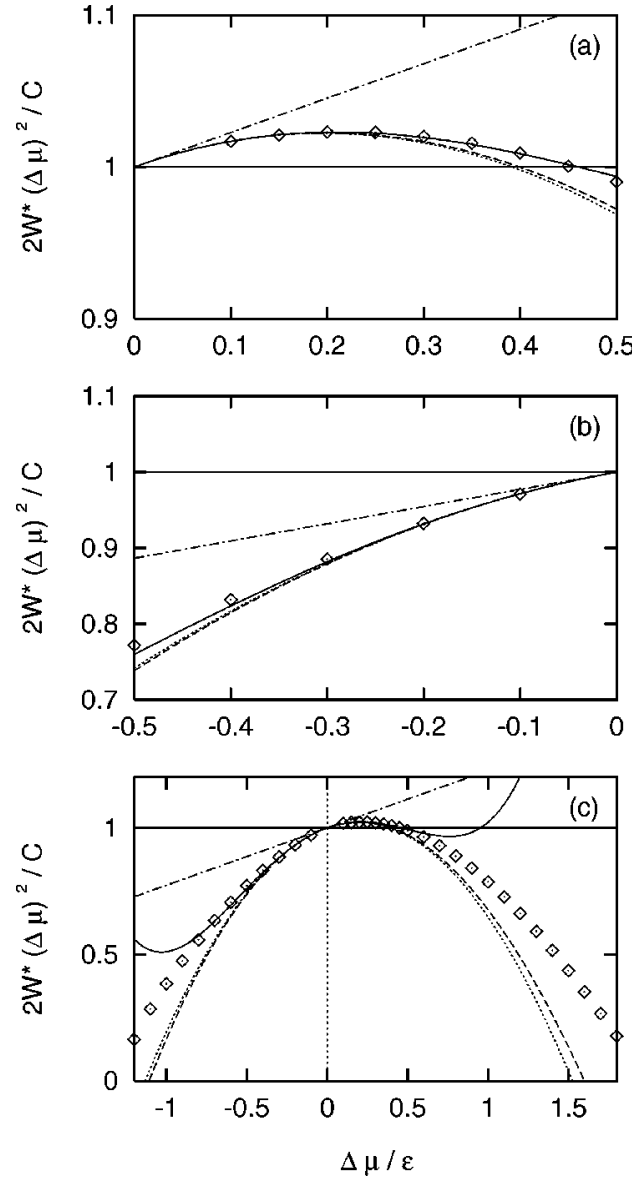

FIG. 1. Nucleation free-energy barrier height $W^{*}$ multiplied by $(\Delta \mu)^{2} / C$ : the density-functional result for the Lennard-Jones critical nucleus (black circles), the classical nucleation theory (the horizontal lines), first (dasheddotted lines), second (dotted curves), third (dashed curves) and fourth (solid curves) order approximation of the expansion formula. The coefficients in the expansion were obtained from the density functional calculation of the vapor-liquid interface at stable equilibrium and the critical droplets and bubbles. Temperature is 0.7 in reduced units. All the values are in reduced units.

units. Inclusion of the fourth-order term gives rise to an excellent agreement with the result of DF calculation within the range: $\left|\Delta \mu^{*}\right| \leqslant 0.5$ as shown by the solid curves in Figs. 1(a) and 1(b). However deviation from the DF result increases rapidly in the range of large $\Delta \mu$. The scaling relation for $W^{*}$ proposed by McGraw and Laaksonen does not have the term with coefficient $B$ but does include the important term with coefficient $D$. This is the main reason why the scaling relation with a single parameter $D$ (beside $C$ ) significantly improves CNT prediction over a wide range of $\Delta \mu$. However in order to account for the asymmetrical feature of $2 W^{*}(\Delta \mu)^{2} / C$, the inclusion of the term with coefficient $B$ is essential.

The expansion formula in Eq. (22) for $\Delta n^{*}$ is also examined through comparison with the DF result. The quantity $\Delta n^{*}(\Delta \mu)^{3} / C$ is plotted against $\Delta \mu$ in reduced unit in Fig. 2. It is found that $\Delta n^{*}(\Delta \mu)^{3}$ varies only $15 \%$ over a whole range of $\Delta \mu$. Therefore, the zeroth order approximation for $\Delta n^{*}$ (the Kelvin relation) corresponding to the horizontal lines in Fig. 2 is a good approximation beyond a small range close to $\Delta \mu=0$. This is in contrast to the zeroth-order ap-
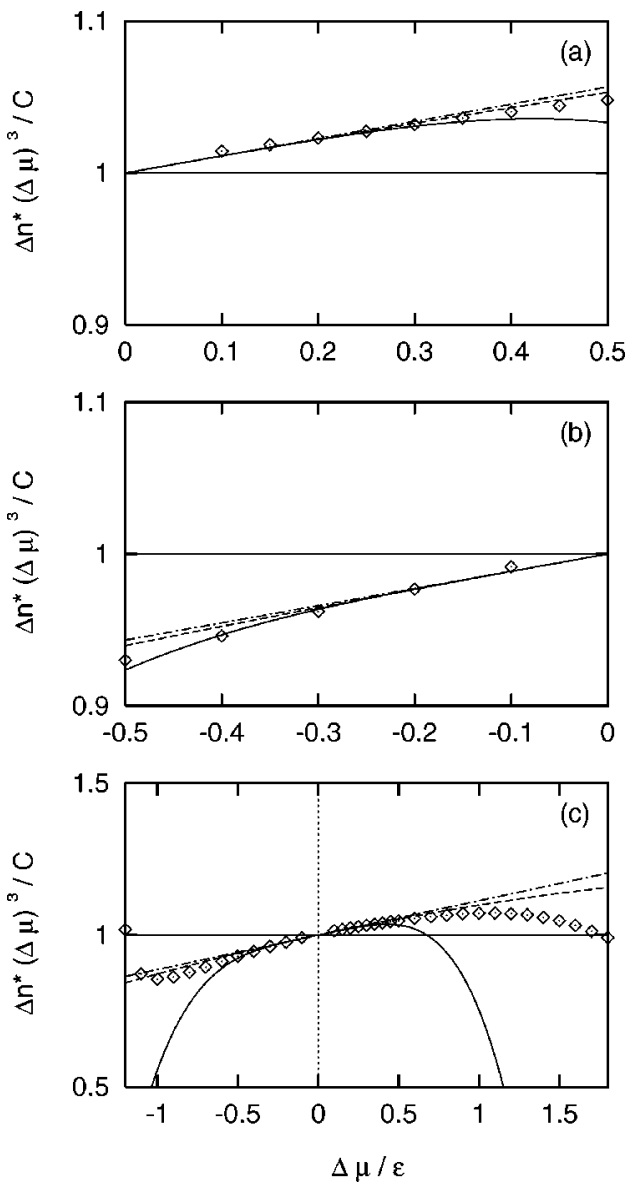

FIG. 2. Size of the critical nucleus $\Delta n^{*}$ multiplied by $(\Delta \mu)^{3} / C$ : the density-functional result (black circles), the classical nucleation theory (the horizontal lines), first (dashed-dotted lines), third (dashed curves), and fourth (solid curves) order approximation of the expansion formula. The conditions are the same as in Fig. 1. All the values are in reduced units.

proximation $(\mathrm{CNT})$ for $W^{*}$. Introduction of the first-order term with coefficient $B$ improves further the classical prediction and gives the correct slope at $\Delta \mu=0$. Improvement made by the third-order term with coefficient $A_{1}$ is found to be insignificant as in the case for $W^{*}$. The expansion up to the fourth-order term with coefficient $A_{2}$ betters the result in a certain range up to \pm 0.4 [Figs. 2(a) and 2(b)], but gives rise to large errors for large $\Delta \mu$. These results show that the scaling relation for $\Delta \mu$, i.e., extension of the Kelvin relation to a wide range of $\Delta n^{*}$, is reasonable though inexact. The other scaling relations are also successful because they are derived from the scaling relation for $\Delta n^{*}$.

\section{CONCLUSION}

The nucleation free-energy barrier height $W^{*}$ and the size of the critical nucleus $\Delta n^{*}$ are expressed in powers of the chemical potential difference $\Delta \mu$ from the stable equilibrium state. The coefficients in the thermodynamic expansion are written in terms of the bulk and surface properties at the stable equilibrium state of the vapor-liquid coexistence. Through comparisons with the density functional results, we have observed systematic improvement of the prediction of the expansion formulas as adding the higher order terms both for $W^{*}$ and $\Delta n^{*}$. We have found that introduction of the 
second order term with coefficient $D$ is crucial to describe the parabola-like behavior of $W^{*}(\Delta \mu)^{2}$ over a wide range of $\Delta \mu$. Asymmetry in the behavior of $W^{*}(\Delta \mu)^{2}$ between vapor-to-liquid and liquid-to-vapor nucleation is accounted for by the first order term with coefficient $B$, which include the sum of the Tolman length and the Egelstaff and Widom fundamental length characteristic of liquid. For the behavior of $\Delta n^{*}(\Delta \mu)^{3}$, we have found that the zeroth order term alone is a good approximation beyond a small range of $\Delta \mu$. Inclusion of the first order term with coefficient $B$ accounts for the asymmetry in $\Delta n^{*}(\Delta \mu)^{3}$ and gives rise to more precise prediction in a wide range of $\Delta \mu$.

From the general expansion formulas we can recover the scaling relations for the critical nucleus proposed by McGraw and Laaksonen via neglecting the first order coefficient $B$ and the third and higher order coefficients. Furthermore the parameter $D(T)$ in the scaling relations is identified as the second order coefficient in the expansion of $W^{*}$ and can be given explicitly in terms of the thermodynamic properties at the stable equilibrium. The scaling relation for $\Delta n^{*}$, corresponding to the zeroth order approximation to the expansion, is indeed a reasonable approximation for both the droplet and bubble formation. Thus other scaling relations resulted from this relation through the nucleation theorem are also good approximations given that an appropriate value for the parameter $D(T)$ is known. However, the scaling relations do not account for any asymmetry in the behavior of $W^{*}(\Delta \mu)^{2}$ and $\Delta n^{*}(\Delta \mu)^{3}$; instead, they predict complete symmetry of these quantities with respect to $\Delta \mu=0$ and result in $\delta_{\infty}=-\Delta_{\infty} / 3$, a particular relation between the Tolman length and the fundamental length characteristic of the liquid. However, $W^{*}(\Delta \mu)^{2}$ and $\Delta n^{*}(\Delta \mu)^{3}$ obtained from the DF calculation of the LJ fluid exhibit asymmetry between droplet nucleation $(\Delta \mu>0)$ and bubble formation $(\Delta \mu$ $<0$ ), and the relation between $\delta_{\infty}$ and $\Delta_{\infty}$ is rather given by $\delta_{\infty} \approx-\Delta_{\infty}$. Therefore, in general, the first-order term with coefficient $B$ is necessary in order to describe asymmetrical features for the critical nucleus.

\section{ACKNOWLEDGMENTS}

We thank Dr. A. K. Shchekin for fruitful discussions. X.C.Z. thanks NSF for support of this work.

${ }^{1}$ See, for example, A. Laaksonen, V. Talanquer, and D. W. Oxtoby, Annu. Rev. Phys. Chem. 46, 489 (1995).

${ }^{2}$ M. Volmer and A. Weber, Z. Phys. Chem. 119, 277 (1925); R. Becker and W. Döring, Ann. Phys. 24, 719 (1935); J. Frenkel, J. Chem. Phys. 7, 538 (1939).

${ }^{3}$ H. M. Ellerby and H. Reiss, J. Chem. Phys. 97, 5766 (1992); C. L. Weakliem and H. Reiss, J. Chem. Phys. 98, 6408 (1994); C. L. Weakliem and H. Reiss, J. Chem. Phys. 101, 2398 (1994).

${ }^{4}$ D. W. Oxtoby and R. Evans, J. Chem. Phys. 89, 7521 (1988).

${ }^{5}$ X. C. Zeng and D. W. Oxtoby, J. Chem. Phys. 94, 4472 (1991).

${ }^{6}$ V. Talanquer and D. W. Oxtoby, J. Chem. Phys. 100, 5190 (1993).

${ }^{7}$ R. McGraw and A. Laaksonen, Phys. Rev. Lett. 76, 2754 (1996).

${ }^{8}$ R. McGraw and A. Laaksonen, J. Chem. Phys. 106, 5284 (1997).

${ }^{9}$ V. Talanquer, J. Chem. Phys. 106, 9957 (1997).

${ }^{10}$ G. W. Adams, J. L. Schmitt, and R. A. Zalabsky, J. Chem. Phys. 81, 5074 (1984); R. Strey, P. E. Wanger, and T. Schmeling, ibid. 84, 2325 (1986).

${ }^{11}$ We note that if validity of the Kelvin relation beyond the limit $\Delta \mu \rightarrow 0$ is assumed, the McGraw-Laaksonen scaling relations can be also derived without using the homogeneity ansatz.

${ }^{12}$ R. C. Tolman, J. Chem. Phys. 17, 333 (1949).

${ }^{13}$ P. A. Egelstaff and B. Widom, J. Chem. Phys. 53, 2667 (1970).

${ }^{14}$ J. W. Gibbs, The Scientific Papers of J. Willard Gibbs (Ox Bow, Woodbridge, 1993).

${ }^{15}$ J. S. Rowlinson and B. Widom, Molecular Theory of Capillarity (Oxford University, New York, 1989).

${ }^{16}$ D. Kashchiev, J. Chem. Phys. 76, 5098 (1982).

${ }^{17}$ D. W. Oxtoby and D. Kashchiev, J. Chem. Phys. 100, 7665 (1994).

${ }^{18}$ Y. Viisanen, R. Strey, and H. Reiss, J. Chem. Phys. 99, 4680 (1993).

${ }^{19}$ Several polynomials of different order (from 6 to 16) are used to fit a set of data $\left(\Delta \mu, \sigma_{\mathrm{s}}\right)$ in a range of $\Delta \mu$ between -0.5 and 1.2 in reduced units. The third and fourth order fitting parameters which determine $\sigma^{\prime \prime \prime \prime}$ and $\sigma$ are relatively sensitive to the choice of polynomial, compared to the first and second parameters which determine $\sigma^{\prime}$ and $\sigma^{\prime \prime}$. However we confirmed that our conclusions do not depend on the choice of the polynomial. The results given here are obtained by ten-parameter fitting.

${ }^{20}$ K. Koga, X. C. Zeng, and A. K. Shchekin, J. Chem. Phys. 109, 4063 (1998).

${ }^{21}$ There are some theoretical results which imply the close relationship between the Tolman length and the fundamental length. First, within a framework of the gradient theories the Tolman length is given analytically as $\delta_{\infty}=-\Delta_{\infty}$. (Refs. 22, 23) Also DF calculation of LJ and Yukawa model fluids shows that the relation, $\delta_{\infty}(T)=-k \Delta_{\infty}(T)$ where $k$ is a constant close to 1 , holds at least for a range of $T$ near the triple point (Ref. 23).

${ }^{22}$ M. Iwamatsu, J. Phys.: Condens. Matter 6, L173 (1994).

${ }^{23}$ T. V. Bykov and A. K. Shchekin (unpublished). 